\title{
THE NILPOTENCY OF BANACH NIL ALGEBRAS
}

\section{SANDY GRABINER}

THEOREM. If $B$ is a Banach algebra which is also a nil algebra, then $B$ is a nilpotent algebra.

Proof. For each positive integer $j$, define

$$
N_{j}=\left\{x \in B: x^{j}=0\right\} .
$$

By the theorem of Nagata-Higman [1, p. 274], it will be enough to show that some $N_{j}=B$. Each $N_{j}$ is closed and the union of the $N_{j}$ is $B$. Hence, by the Baire Category Theorem, there is a fixed integer $k$ and a fixed $z \in B$ for which $N_{k}$ is a neighborhood of $z$. Suppose $x \in B$. Define the $B$ valued polynomial of a scalar variable $t, p(t)$ $=(z+t(x-z))^{k}$. Since $z+t(x-z)$ is continuous, $p(t)$ must equal zero for all sufficiently small $t$. Therefore, $p(t) \equiv 0$; and, in particular $p(1)$ $=x^{k}=0$. This completes the proof.

It is easy to construct examples of normed algebras for which the above theorem is false, though the theorem is true for second category topological algebras.

\section{REFERENCE}

1. N. Jacobson, Structure of rings, rev. ed., Amer. Math Soc. Colloq. Publ., Vol. 37, Amer. Math. Soc., Providence, R. I., 1964.

Massachusetts Institute of Technology

Received by the editors August 9, 1968. 